\title{
THE CAUCHY PROBLEM FOR NONLINEAR ELLIPTIC EQUATIONS
}

\author{
I. LY AND N. TARKHANOV
}

Abstract. This paper is devoted to investigation of the Cauchy problem for nonlinear elliptic equations with a small parameter.

\section{Contents}

1. Introduction

2. An example

3. The Cauchy problem

4. Reduction

5. The Dirichlet problem

6. Strong solutions

7. Nonzero boundary data

8. The Poisson formula

9. Nonlinear perturbation

10. The Cauchy problem revisited - 10

11. The Zaremba problem 11

12. Linearisable problems 12

References

\section{INTRODUCTION}

The question of the well-posedness of the Cauchy problem was first raised by J. Hadamard who proved that it is ill-posed in the case of linear second order elliptic equations. But the introduction in $[\operatorname{Had} 27]$ clearly indicates that Hadamard was interested in nonlinear equations as well. In modern words, Hadamard's proof is based on the analytic regularity of solutions to linear elliptic boundary value problems. This regularity has been extended to nonlinear elliptic equations by Morrey [Mor58], so that Hadamard's argument also applies to general nonlinear elliptic equations.

The Cauchy problem for elliptic equations still remains a challenge for modern analysis, cf. [Tar95]. This problem, being ill-posed, cannot be handled within any nowadays available operator algebra. Together with Fredholm integral equations of the first kind it initiated the development of a new area in the 1950s, called the theory of inverse problems.

In [ST05] the Cauchy problem for elliptic equations is specified in a class of mixed boundary value problems depending on a small parameter. A calculus of parameter-dependent mixed boundary value problems can be got by 'edgification' of Boutet de Monvel's algebra.

By the very nature, an elliptic problem with parameter is invertible if the parameter is large enough. In contrast to this, the Cauchy problem corresponds to

2000 Mathematics Subject Classification. Primary 35J65; Secondary 34G20.

Key words and phrases. Nonlinear equations, ellipticity, Cauchy problem. 
the parameter going to zero. Of course, this idea is not new in mathematics and it goes back at least as far as L. Prandtl. It is explicit in [Mas68] and actually makes the contents of [TA79].

Methods of [Tar95] and [ST05] go certainly beyond the linear partial differential equations and shed some new light on the Cauchy problem for nonlinear elliptic equations. With this as our starting point, we examine in this paper to what extent mixed boundary value problems of Zaremba type may be of use to study the nonlinear Cauchy problem.

As but easy consequences of our approach we mention that the Cauchy problem is normally solvable if the Cauchy data are given on the whole boundary. Moreover, we elucidate the unstable character of this problem if the Cauchy data are carried by a part of boundary.

\section{AN EXAMPLE}

Let $F(y)$ be a differentiable function of $y \in(-R, R)$, where $R>0$. Consider the initial problem

$$
\begin{aligned}
F^{\prime}(y) y^{\prime} & =1 \quad \text { in } \quad(0,1) \\
y(0) & =y_{0}
\end{aligned}
$$

for a function $y=y(x)$ on the interval $(0,1) \subset \mathbb{R}$. The initial value $y_{0}$ is assumed to belong to $(-R, R)$.

The first integral of the ordinary differential equation is $F(y)=x+c$, with $c$ an arbitrary constant. The initial condition yields $F\left(y_{0}\right)=c$, hence $(2.1)$ reduces to $F(y)=x+F\left(y_{0}\right)$.

Thus, the question on solvability and uniqueness for the quasilinear initial problem is equivalent to that for the functional equation $F(y)=x+F\left(y_{0}\right)$. This latter possesses in general no satisfactory answer. The implicit function theorem gives conditions towards existence of a local solution $y=y(x)$, namely $F^{\prime}(y) \neq 0$ for all $y \in(-R, R)$, which just amounts to the ellipticity of the differential equation $F^{\prime}(y) y^{\prime}=1$.

In general, there are no reasonable conditions both necessary and sufficient for the existence of a global solution $y=y(x)$ to $F(y)=x+F\left(y_{0}\right)$ on the interval $(0,1)$. In order to get asymptotic results, it is therefore necessary to put some restrictions on $F$. If e.g. $F(y)=-\exp y$, then $y(x)=\log \left(\exp y_{0}-x\right)$, and so the initial value should satisfy $y_{0} \geq 0$.

\section{The Cauchy problem}

Since any system of partial differential equations can be reduced by introducing new unknown functions to a first order system, we restrict our attention to these latter.

Let $A$ be an $(\ell \times k)$-matrix of first order linear scalar partial differential operators with smooth coefficients on an open set $\mathcal{X}$ in $\mathbb{R}^{n}$. We assume that the principal homogeneous symbol of $A$ has rank $k$ at each point $(x, \xi) \in T^{*} \mathcal{X}$ with $\xi \neq 0$. This is equivalent to saying that the differential operator $\Delta=A^{*} A$ called the Laplacian of $A$ is elliptic of order 2. Here, $A^{*}$ is the formal adjoint of $A$. If $\ell=k$ then $A$ itself is elliptic.

Given a domain $\mathcal{D} \Subset \mathcal{X}$ with smooth boundary and a non-empty open piece $\mathcal{S} \subset \partial \mathcal{D}$ with smooth boundary, we consider the Cauchy problem

$$
\begin{aligned}
A y & =\varepsilon f\left(x, y, y^{\prime}\right) & \text { in } \mathcal{D}, \\
y & =y_{0} & \text { on } \mathcal{S},
\end{aligned}
$$

where $y^{\prime}$ stands for the Jacobi matrix of $y, f(x, y, p)$ is a function on $\mathcal{D} \times \mathbb{C}^{k} \times \mathbb{C}^{k \times n}$ with values in $\mathbb{C}^{\ell}, u_{0}$ a function on $\mathcal{S}$ with values in $\mathbb{C}^{k}$, called Cauchy data, and $\varepsilon$ a small parameter. 
The function $f(x, y, p)$ is assumed to belong to the class $L^{q}\left(\mathcal{D}, \mathbb{C}^{\ell}\right)$ in $x$ and satisfy a Lipschitz condition in $y$ and $p$. The Lipschitz constants of $f$ with respect to $y$ and $p$ are denoted by $\Lambda_{y} f$ and $\Lambda_{p} f$, respectively, and assumed to be uniformly bounded in $x \in \mathcal{D}$.

When dealing with nonlinear differential equations in Sobolev spaces, one uses the following result which works over $\mathbb{R}^{n}$ as well as compact manifolds.

Theorem 3.1. Let " $\times$ " denote any bilinear map. Then:

1) Pointwise application of $\times$ induces a continuous map from $W^{s, q} \times W^{t, q}$ to $L^{q}$ if $s+t>n / q$.

2) If $s>n / q$ and $0 \leq t \leq s$ then pointwise application of $\times$ induces a continuous map from $W^{s, q} \times W^{t, q}$ to $W^{t, q}$.

Proof. See for instance [Pal68].

The natural Sobolev space setting for the study of Cauchy problem (3.1) is certainly $u \in W^{1, q}\left(\mathcal{D}, \mathbb{C}^{k}\right)$, where $q \geq 1$ is at least as large as $1>n / q$. By the Sobolev embedding theorem, the Cauchy data $u_{0}$ are to be chosen in the space $W^{1-1 / q, q}\left(\mathcal{S}, \mathbb{C}^{k}\right)$.

The unperturbed Cauchy problem corresponding to $\varepsilon=0$ is linear, and so illposed unless $\mathcal{S}=\partial \mathcal{D}$, see [Tar95]. In this latter case the unperturbed Cauchy problem is normally solvable, however, the space of those data for which the problem is solvable has infinite codimension. Hence, small perturbations of the right-hand side can lead out of the space of admissible data, and so the standard perturbation theory does not apply.

\section{REDUCTION}

Our goal is to invoke the Dirichlet problem for studying (3.1), since the Dirichlet problem is the best understood elliptic boundary value problem. To this end, we note that both $A y$ and $f\left(x, y, y^{\prime}\right)$ are $L^{q}$-functions in $\mathcal{D}$, for $f(x, y, p)$ is Lipschitzian in $y$ and $p$. Therefore, we may apply the linear differential operator $A^{*}$ to both sides of the differential equation in (3.1), thus obtaining $\Delta y=\varepsilon A^{*} f\left(x, y, y^{\prime}\right)$ in $\mathcal{D}$. Note that the formal adjoint $A^{*}$ maps $L^{q}\left(\mathcal{D}, \mathbb{C}^{\ell}\right)$ continuously to $W^{-1, q}\left(\mathcal{D}, \mathbb{C}^{k}\right)$, the dual of

$$
W^{1, q^{\prime}}\left(\mathcal{D}, \mathbb{C}^{k}\right)
$$

with respect to the $L^{2}\left(\mathcal{D}, \mathbb{C}^{k}\right)$-pairing, $1 / q+1 / q^{\prime}=1$

When substituting $\Delta y=\varepsilon A^{*} f\left(x, y, y^{\prime}\right)$ for $A y=\varepsilon f\left(x, y, y^{\prime}\right)$, one leaves behind the genuine Cauchy problem. In order to provide the equivalence we shall keep a trace of the equality $A y=\varepsilon f\left(x, y, y^{\prime}\right)$ on $\partial \mathcal{D}$. For $y \in W^{1, q}\left(\mathcal{D}, \mathbb{C}^{k}\right)$, the difference $u=A y-\varepsilon f\left(x, y, y^{\prime}\right)$ belongs to the space $L^{q}\left(\mathcal{D}, \mathbb{C}^{\ell}\right)$. In the scale of usual Sobolev spaces it no longer possesses any restriction to the boundary of $\mathcal{D}$. However, the equality $u=0$ on $\partial \mathcal{D}$ can be interpreted in a weak sense. We are thus led to the boundary problem

cf. (3.1).

$$
\begin{aligned}
\Delta y & =\varepsilon A^{*} f\left(x, y, y^{\prime}\right) & & \text { in } \mathcal{D}, \\
y & =y_{0} & & \text { on } \mathcal{S}, \\
A y & =\varepsilon f\left(x, y, y^{\prime}\right) & & \text { on } \partial \mathcal{D},
\end{aligned}
$$

Theorem 4.1. Suppose $A^{*} u=0$ has no non-trivial solutions on $\mathcal{X}$ with support in $\overline{\mathcal{D}}$. Then $y \in W^{1, q}\left(\mathcal{D}, \mathbb{C}^{k}\right)$ is a solution of (3.1) if and only if it satisfies (4.1).

Proof. The necessity is obvious, it suffices to prove the sufficiency. For this purpose, set $u=A y-\varepsilon f\left(x, y, y^{\prime}\right)$. Then $u \in L^{q}\left(\mathcal{D}, \mathbb{C}^{\ell}\right)$. Since $A^{*} u=0$ in $\mathcal{D}$ and $u=0$ on $\partial \mathcal{D}$, it follows that the function $\tilde{u}: \mathcal{X} \rightarrow \mathbb{C}^{\ell}$, which is equal to $u$ in $\mathcal{D}$ and to 0 in $\mathcal{X} \backslash \mathcal{D}$, satisfies $A^{*} \tilde{u}=0$ in all of $\mathcal{X}$. Hence, $\tilde{u}=0$ in $\mathcal{X}$, and so $u=0$ in $\mathcal{D}$, as desired. 
The hypothesis of Theorem 4.1 is fulfilled, in particular, if $A$ is elliptic (i.e., $\ell=k$ ) and has real-analytic coefficients in $\mathcal{X}$. If $\varepsilon=0$, then the condition $\ell=k$ can be omitted, cf. [Tar95, Theorem 10.3.11].

\section{The Dirichlet Problem}

Given a vector space $V$ with norm $\|\cdot\|$, we write $C(V,\|\cdot\|)$ for the completion of $V$ under the norm $\|\cdot\|$.

Our next goal is to describe the Hodge theory of the Dirichlet problem for the Laplacian $\Delta=A^{*} A$ in the class of generalised functions on $\mathcal{D}$. In order to define what is meant by the "solution" of the boundary value problem, we employ negative norms. This idea is certainly not new and goes back at least as far as [Sch60] and [Roi96].

Pick a Dirichlet system $B$ of order 0 on the boundary of $\mathcal{D}$. This is actually an invertible $(k \times k)$-matrix of smooth functions in a neighbourhood $U$ of $\partial \mathcal{D}$.

Let $C$ be the adjoint system for $B$ with respect to Green's formula for the Laplace operator $\Delta$, cf. [Tar95]. Thus, $C$ is an $(\ell \times k)$-matrix of smooth functions in a smaller neighbourhood $U$ of $\partial \mathcal{D}$. We now set

$$
\begin{aligned}
t(y) & =B y, \\
n(u) & =C^{*} u
\end{aligned}
$$

for $y \in C\left(U, \mathbb{C}^{k}\right)$ and $u \in C\left(U, \mathbb{C}^{\ell}\right)$.

Lemma 5.1 (Green's formula). For all $y \in W^{2, q}\left(\mathcal{D}, \mathbb{C}^{k}\right)$ and $z \in W^{2, q^{\prime}}\left(\mathcal{D}, \mathbb{C}^{k}\right)$ the following formula holds:

$$
\int_{\partial \mathcal{D}}\left((t(y), n(A z))_{x}-(n(A y), t(z))_{x}\right) d s=\int_{\mathcal{D}}\left((\Delta y, z)_{x}-(y, \Delta z)_{x}\right) d x .
$$

Proof. Cf. Corollary 9.2.12 in [Tar95].

Given $w$, we consider the boundary value problem

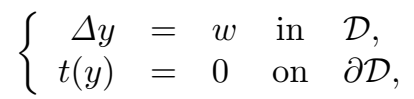

which is an obvious generalisation of the classical Dirichlet problem, cf. 9.2.4 in [Tar95].

Suppose $s>0$ and $1<q<\infty$. For functions $y \in C^{\infty}\left(\overline{\mathcal{D}}, \mathbb{C}^{k}\right)$ we define two types of negative norms

$$
\begin{aligned}
\|y\|_{-s, q} & =\sup _{z \in C^{\infty}\left(\overline{\mathcal{D}}, \mathbb{C}^{k}\right)} \frac{|(y, z)|}{\|z\|_{s, q^{\prime}}}, \\
|y|_{-s, q} & =\sup _{\substack{z \in C^{\infty}\left(\overline{\mathcal{D}}, \mathbb{C}^{k}\right) \\
t(z)=0}} \frac{|(y, z)|}{\|z\|_{s, q^{\prime}}},
\end{aligned}
$$

where $(\cdot, \cdot)$ is the scalar product in $L^{2}\left(\mathcal{D}, \mathbb{C}^{k}\right)$. We denote the completions of $C^{\infty}\left(\overline{\mathcal{D}}, \mathbb{C}^{k}\right)$ with respect to these norms by $W^{-s, q}\left(\mathcal{D}, \mathbb{C}^{k}\right)$ and $C\left(C^{\infty}\left(\overline{\mathcal{D}}, \mathbb{C}^{k}\right),|\cdot|_{-s, q}\right)$, respectively. They are obviously Banach spaces and satisfy

$$
W^{-s, q}\left(\mathcal{D}, \mathbb{C}^{k}\right) \hookrightarrow C\left(C^{\infty}\left(\overline{\mathcal{D}}, \mathbb{C}^{k}\right),|\cdot|_{-s, q}\right),
$$

for $\|y\|_{-s, q} \geq|y|_{-s, q}$.

We can define $(y, z)$ for $y \in W^{-s, q}\left(\mathcal{D}, \mathbb{C}^{k}\right)$ and $z \in C^{\infty}\left(\overline{\mathcal{D}}, \mathbb{C}^{k}\right)$ as follows. By definition, there is a sequence $\left\{y_{\nu}\right\}$ in $C^{\infty}\left(\overline{\mathcal{D}}, \mathbb{C}^{k}\right)$ such that $\left\|y_{\nu}-y\right\|_{-s, q} \rightarrow 0$ as $\nu \rightarrow \infty$. Then

$$
\begin{aligned}
\left|\left(y_{\nu}-y_{\mu}, z\right)\right| & \leq\left\|y_{\nu}-y_{\mu}\right\|_{-s, q}\|z\|_{s, q^{\prime}} \\
& \rightarrow 0
\end{aligned}
$$


as $\mu, \nu \rightarrow \infty$. Set $(y, z)=\lim \left(y_{\nu}, z\right)$. Clearly, this limit does not depend on the particular sequence $\left\{y_{\nu}\right\}$, for if $\left\|y_{\nu}\right\|_{-s, q} \rightarrow 0$ then $\left|\left(y_{\nu}, z\right)\right| \leq\left\|y_{\nu}\right\|_{-s, q}\|z\|_{s, q^{\prime}}$ tends to zero, too. From the definition it follows that for all $y \in W^{-s, q}\left(\mathcal{D}, \mathbb{C}^{k}\right)$ and $z \in C^{\infty}\left(\overline{\mathcal{D}}, \mathbb{C}^{k}\right)$ we get

$$
|(y, z)| \leq\|y\|_{-s, q}\|z\|_{s, q^{\prime}} .
$$

In a similar way we can define the pairing $(y, z)$ for $y \in C\left(C^{\infty}\left(\overline{\mathcal{D}}, \mathbb{C}^{k}\right),|\cdot|{ }_{-s, q}\right)$ and $z \in C^{\infty}\left(\overline{\mathcal{D}}, \mathbb{C}^{k}\right)$ with $t(z)=0$. Corresponding to (5.2) we have obviously $|(y, z)| \leq|y|_{-s, q}\|z\|_{s, q^{\prime}}$.

\section{Strong solutions}

Let $w$ be in $C\left(C^{\infty}\left(\overline{\mathcal{D}}, \mathbb{C}^{k}\right),|\cdot|_{-s-2, q}\right)$, where $s \geq 0$. We say that $y \in W^{-s, q}\left(\mathcal{D}, \mathbb{C}^{k}\right)$ is a strong solution of (5.1) if there is a sequence $y_{\nu} \in C^{\infty}\left(\overline{\mathcal{D}}, \mathbb{C}^{k}\right)$ with $t\left(y_{\nu}\right)=0$, such that

$$
\begin{aligned}
\left\|y_{\nu}-y\right\|_{-s, q} & \rightarrow 0 \\
\left|\Delta y_{\nu}-w\right|_{-s-2, q} & \rightarrow 0
\end{aligned}
$$

as $\nu \rightarrow \infty$.

Denote by $\mathcal{H}(\mathcal{D})$ the set of all $y \in C^{\infty}\left(\overline{\mathcal{D}}, \mathbb{C}^{k}\right)$ that satisfy $\Delta y=0$ in $\mathcal{D}$ and $t(y)=0$ on the boundary of $\mathcal{D}$. Since (5.1) is an elliptic boundary value problem, the space $\mathcal{H}(\mathcal{D})$ is finite dimensional. Moreover, for any $y \in \mathcal{H}(\mathcal{D})$ we actually obtain

$$
\begin{aligned}
0 & =(\Delta y, y) \\
& =(A y, A y)
\end{aligned}
$$

whence $A y=0$ in $\mathcal{D}$. Therefore, the space $\mathcal{H}(\mathcal{D})$ consists of all solutions to $A y=0$ in $\mathcal{D}$, which are $C^{\infty}$ up to the boundary of $\mathcal{D}$ and which vanish up to the infinite order on $\partial \mathcal{D}$.

Lemma 6.1. Let $s \geq 0$ and $1<q<\infty$. If $w \in C\left(C^{\infty}\left(\overline{\mathcal{D}}, \mathbb{C}^{k}\right),|\cdot|_{-s-2, q}\right)$ and $w \perp \mathcal{H}(\mathcal{D})$, then there is a strong solution $y \in W^{-s, q}\left(\mathcal{D}, \mathbb{C}^{k}\right)$ of (5.1) satisfying $y \perp \mathcal{H}(\mathcal{D})$ and

$$
\|y\|_{-s, q} \leq c|w|_{-s-2, q},
$$

where the constant $c$ does not depend on $w$ and $y$.

Proof. Cf. [Sch60].

The definition (6.1) of a strong solution to the Dirichlet problem (5.1) obviously corresponds to an appropriate closure $L: \mathcal{D}_{L} \rightarrow C\left(C^{\infty}\left(\overline{\mathcal{D}}, \mathbb{C}^{k}\right),|\cdot|_{-s-2, q}\right)$ of the Laplacian $\Delta=A^{*} A$, cf. Chapter 2 in [Dez80]. Namely, we denote by $\mathcal{D}_{L}$ the set of all sections $y \in W^{-s, q}\left(\mathcal{D}, \mathbb{C}^{k}\right)$, for which there is a sequence $\left\{y_{\nu}\right\}$ with the following properties:

1) $y_{\nu} \in C^{\infty}\left(\overline{\mathcal{D}}, \mathbb{C}^{k}\right)$ satisfies $t\left(y_{\nu}\right)=0$

2) $\left\{y_{\nu}\right\}$ converges to $y$ in $W^{-s, q}\left(\mathcal{D}, \mathbb{C}^{k}\right)$; and

3) $\left\{\Delta y_{\nu}\right\}$ is a Cauchy sequence in $C\left(C^{\infty}\left(\overline{\mathcal{D}}, \mathbb{C}^{k}\right),|\cdot|_{-s-2, q}\right)$.

The closed densely defined operator $L: \mathcal{D}_{L} \rightarrow C\left(C^{\infty}\left(\overline{\mathcal{D}}, \mathbb{C}^{k}\right),|\cdot|_{-s-2, q}\right)$ given by $L y=\lim \Delta y_{\nu}$, where $\left\{y_{\nu}\right\}$ is any sequence with properties 1$\left.)-3\right)$, is called the strong extension of $\Delta$ under the boundary conditions $t(y)=0$. It is clear that $y \in W^{-s, q}\left(\mathcal{D}, \mathbb{C}^{k}\right)$ is a strong solution to the Dirichlet problem (5.1) if and only if $L y=w$.

It is worth pointing out that the case $\partial \mathcal{D}=\emptyset$ is formally permitted in the following theorem.

Theorem 6.2. Suppose $s \geq 0$. There are bounded linear operators

$$
\begin{aligned}
& H: C\left(C^{\infty}\left(\overline{\mathcal{D}}, \mathbb{C}^{k}\right),|\cdot|_{-s-2, q}\right) \rightarrow \mathcal{H}(\mathcal{D}), \\
& G: C\left(C^{\infty}\left(\overline{\mathcal{D}}, \mathbb{C}^{k}\right),|\cdot|_{-s-2, q}\right) \rightarrow \mathcal{D}_{L}
\end{aligned}
$$


such that

1) $H$ has the kernel $K_{H}\left(x, x^{\prime}\right)=\sum_{\nu} h_{\nu}(x) \otimes h_{\nu}\left(x^{\prime}\right)^{*}$ where $\left\{h_{\nu}\right\}$ is an orthonormal basis of $\mathcal{H}(\mathcal{D})$;

2) $A H=0$ and $G H=H G=0$;

3)

$$
\begin{aligned}
G L y & =y-H y \quad \text { for all } \quad y \in \mathcal{D}_{L} \\
L G w & =w-H w \quad \text { for all } \quad w \in C\left(C^{\infty}\left(\overline{\mathcal{D}}, \mathbb{C}^{k}\right),|\cdot|_{-s-2, q}\right) .
\end{aligned}
$$

Proof. This follows by the same method as in Theorem 3.3 of [SST00], with Lemma 3.2 therefrom replaced by Lemma 6.1 .

The operators $H$ and $G$ are actually independent of $s$ and $q$ since they are unique extensions by continuity of these operators on the dense subspace $C^{\infty}\left(\overline{\mathcal{D}}, \mathbb{C}^{k}\right)$ of $C\left(C^{\infty}\left(\overline{\mathcal{D}}, \mathbb{C}^{k}\right),|\cdot|_{-s-2, q}\right)$.

The action of $G$ on the Hilbert space $L^{2}\left(\mathcal{D}, \mathbb{C}^{k}\right)$ is selfadjoint, and this property can be traced in the general setting. More precisely, given any $w \in L^{q}\left(\mathcal{D}, \mathbb{C}^{k}\right)$ and $z \in L^{q^{\prime}}\left(\mathcal{D}, \mathbb{C}^{k}\right)$, we may invoke the elliptic regularity of the Dirichlet problem (5.1) to see that $G w \in W^{2, q}\left(\mathcal{D}, \mathbb{C}^{k}\right)$ and $G z \in W^{2, q^{\prime}}\left(\mathcal{D}, \mathbb{C}^{k}\right)$, and they satisfy the boundary conditions $t(G w)=0$ and $t(G z)=0$, respectively. It follows that $L G w=\Delta G w$ and $L G z=\Delta G z$ whence

$$
\begin{aligned}
(G w, z) & =(G w, H z+L G z) \\
& =\left(G w, A^{*} A G z\right) \\
& =\left(A^{*} A G w, G z\right) \\
& =(w, G z)
\end{aligned}
$$

which is due to Theorem 6.2. Hence the Schwartz kernel of $G$ is Hermitean, i.e., $K_{G}\left(x, x^{\prime}\right)^{*}=K_{G}\left(x^{\prime}, x\right)$ for all $\left(x, x^{\prime}\right)$ away from the diagonal of $\overline{\mathcal{D}} \times \overline{\mathcal{D}}$.

Corollary 6.3. If, in addition, $w \in W^{-s-2, q}\left(\mathcal{D}, \mathbb{C}^{k}\right)$, then there is a sequence of sections $y_{\nu} \in C^{\infty}\left(\overline{\mathcal{D}}, \mathbb{C}^{k}\right)$ with $t\left(y_{\nu}\right)=0$, such that

$$
\begin{aligned}
\left\|y_{\nu}-y\right\|_{-s, q} & \rightarrow 0 \\
\left\|\Delta y_{\nu}-w\right\|_{-s-2, q} & \rightarrow 0
\end{aligned}
$$

as $\nu \rightarrow \infty$.

From Lemma 5.1 we deduce that when $y$ is smooth enough, it fulfills $t(y)=0$ if and only if $(\Delta y, z)=(y, \Delta z)$ for all $z$ satisfying $t(z)=0$. This gives rise to the concept of a weak extension of the Laplacian $\Delta$ under the boundary conditions $t(y)=0$, cf. Chapter 2 in [Dez80]. Given any $w \in C\left(C^{\infty}\left(\overline{\mathcal{D}}, \mathbb{C}^{k}\right),|\cdot|_{-s-2, q}\right)$, a section $y$ is said to be a weak solution of $(5.1)$ if it is in $W^{-s^{\prime}, q}\left(\mathcal{D}, \mathbb{C}^{k}\right)$ for some $s^{\prime} \geq 0$ and

$$
(y, \Delta z)=(w, z)
$$

for all $z \in C^{\infty}\left(\overline{\mathcal{D}}, \mathbb{C}^{k}\right)$ satisfying $t(z)=0$.

Lemma 6.4. Suppose that $w \in C\left(C^{\infty}\left(\overline{\mathcal{D}}, \mathbb{C}^{k}\right),|\cdot|_{-s-2, q}\right)$ where $s \geq 0,1<q<\infty$. If $y \in W^{-s^{\prime}, q}\left(\mathcal{D}, \mathbb{C}^{k}\right)$ is a weak solution of $(5.1)$, then actually $y \in W^{-s, q}\left(\mathcal{D}, \mathbb{C}^{k}\right)$ and it is a strong solution of (5.1). Moreover, there is a constant $c$ not depending on $w$ or $y$, such that

$$
\|y\|_{-s, q} \leq c\left(|w|_{-s-2, q}+\|y\|_{-s^{\prime}, q}\right) .
$$

Proof. Cf. [Sch60]. 


\section{NONZERO BOUNDARY DATA}

To study the Dirichlet problem with nonzero boundary data $t(y)=y_{0}$ we need a result of $\left[\right.$ Roi96]. Denote by $W^{-s, q ; B}\left(\mathcal{D}, \mathbb{C}^{k}\right)$ the completion of $C^{\infty}\left(\overline{\mathcal{D}}, \mathbb{C}^{k}\right)$ with respect to the norm

$$
\|y\|_{-s, q ; B}:=\|y\|_{-s, q}+\|t(y)\|_{W^{-s-1 / q, q}\left(\partial \mathcal{D}, \mathbb{C}^{k}\right)}+\|n(A y)\|_{W^{-s-1-1 / q, q}\left(\partial \mathcal{D}, \mathbb{C}^{k}\right)} .
$$

The advantage of using these spaces is that for each $y \in W^{-s, q ; B}\left(\mathcal{D}, \mathbb{C}^{k}\right)$ there is a sequence $\left\{y_{\nu}\right\}$ in $C^{\infty}\left(\overline{\mathcal{D}}, \mathbb{C}^{k}\right)$, such that $y_{\nu} \rightarrow y$ in $W^{-s, q}\left(\mathcal{D}, \mathbb{C}^{k}\right)$, and $\left\{t\left(y_{\nu}\right)\right\}$, $\left\{n\left(A y_{\nu}\right)\right\}$ are Cauchy sequences in $W^{-s-1 / q, q}\left(\partial \mathcal{D}, \mathbb{C}^{k}\right)$ and $W^{-s-1-1 / q, q}\left(\partial \mathcal{D}, \mathbb{C}^{k}\right)$, respectively. Moreover, $\left\{\Delta y_{\nu}\right\}$ is a Cauchy sequence in $W^{-s-2, q}\left(\mathcal{D}, \mathbb{C}^{k}\right)$, which readily follows by manipulations of Green's formula. Therefore, to any element $y \in W^{-s, q ; B}\left(\mathcal{D}, \mathbb{C}^{k}\right)$ we can assign both $t(y), n(A y)$ and $\Delta y$ defined in the above strong sense.

Lemma 7.1. For each pair

$$
\begin{aligned}
& y_{0} \in W^{-s-1 / q, q}\left(\partial \mathcal{D}, \mathbb{C}^{k}\right), \\
& y_{1} \in W^{-s-1-1 / q, q}\left(\partial \mathcal{D}, \mathbb{C}^{k}\right),
\end{aligned}
$$

there is a function $y \in W^{-s, q ; B}\left(\mathcal{D}, \mathbb{C}^{k}\right)$ satisfying $t(y)=y_{0}$ and $n(A y)=y_{1}$. Moreover, the mapping $\left(y_{0}, y_{1}\right) \mapsto y$ is continuous in the relevant norms.

Proof. See Lemma 6.1.2 in [Roi96].

Given any

$$
\begin{aligned}
w & \in C\left(C^{\infty}\left(\overline{\mathcal{D}}, \mathbb{C}^{k}\right),|\cdot|-s-2, q\right), \\
y_{0} & \in W^{-s-1 / q, q}\left(\partial \mathcal{D}, \mathbb{C}^{k}\right),
\end{aligned}
$$

we now consider the inhomogeneous Dirichlet problem

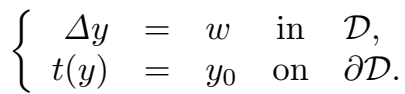

A section $y$ is said to be a weak solution of $(7.2)$ if it is in $W^{-s^{\prime}, q}\left(\mathcal{D}, \mathbb{C}^{k}\right)$ for some $s^{\prime} \geq 0$ and

$$
(y, \Delta z)=(w, z)-\int_{\partial \mathcal{D}}\left(y_{0}, n(A z)\right)_{x} d s
$$

for all $z \in C^{\infty}\left(\overline{\mathcal{D}}, \mathbb{C}^{k}\right)$ satisfying $t(z)=0$.

Theorem 7.2. Suppose $s \geq 0$ and $1<q<\infty$. If $w \perp \mathcal{H}(\mathcal{D})$, then there is a weak solution $y \in W^{-s^{\prime}, q}\left(\mathcal{D}, \mathbb{C}^{k}\right)$ to (7.2) with $y \perp \mathcal{H}(\mathcal{D})$. Moreover, $y \in W^{-s, q}\left(\mathcal{D}, \mathbb{C}^{k}\right)$ satisfies (7.2) in a strong sense, and there is a constant $c$ independent of $w, y_{0}$ and $y$, such that

$$
\|y\|_{-s, q} \leq c\left(|w|_{-s-2, q}+\left\|y_{0}\right\|_{W^{-s-1 / q, q}\left(\partial \mathcal{D}, \mathbb{C}^{k}\right)}\right) .
$$

Proof. Using Lemma 7.1 we reduce (7.3) to (6.4) with a suitable right side $w$. To this end we choose $Y \in W^{-s, q ; B}\left(\mathcal{D}, \mathbb{C}^{k}\right)$ with the property that $t(Y)=y_{0}$ and $n(A Y)=y_{1}, y_{1}$ being arbitrary. By the above, there is a sequence $\left\{y_{\nu}\right\}$ in $C^{\infty}\left(\overline{\mathcal{D}}, \mathbb{C}^{k}\right)$ such that

$$
\begin{aligned}
& y_{\nu} \rightarrow Y \quad \text { in } \quad W^{-s, q}\left(\mathcal{D}, \mathbb{C}^{k}\right), \\
& t\left(y_{\nu}\right) \rightarrow y_{0} \text { in } W^{-s-1 / q, q}\left(\partial \mathcal{D}, \mathbb{C}^{k}\right), \\
& n\left(A y_{\nu}\right) \rightarrow y_{1} \quad \text { in } W^{-s-1-1 / q, q}\left(\partial \mathcal{D}, \mathbb{C}^{k}\right)
\end{aligned}
$$

and $\Delta y_{\nu} \rightarrow w^{\prime}$ in $W^{-s-2, q}\left(\mathcal{D}, \mathbb{C}^{k}\right)$.

By Green's formula, we get

$$
\left(y_{\nu}, \Delta z\right)=\left(\Delta y_{\nu}, z\right)-\int_{\partial \mathcal{D}}\left(t\left(y_{\nu}\right), n(A z)\right)_{x} d s
$$


for all $z \in C^{\infty}\left(\overline{\mathcal{D}}, \mathbb{C}^{k}\right)$ satisfying $t(z)=0$. Letting $\nu \rightarrow \infty$ in this equality yields

$$
(Y, \Delta z)=\left(w^{\prime}, z\right)-\int_{\partial \mathcal{D}}\left(y_{0}, n(A z)\right)_{x} d s .
$$

Subtracting (7.5) from (7.3) we obtain

$$
(y-Y, \Delta z)=\left(w-w^{\prime}, z\right)
$$

for all $z \in C^{\infty}\left(\overline{\mathcal{D}}, \mathbb{C}^{k}\right)$ satisfying $t(z)=0$, i.e., $y-Y$ is a weak solution of the Dirichlet problem (5.1) with $w$ replaced by $w-w^{\prime}$. Moreover, it follows from (7.5) that

$$
\left(w^{\prime}, z\right)=0
$$

for all $z \in \mathcal{H}(\mathcal{D})$. Combining Lemmas 6.4 and 6.1 thus results in the desired assertion.

\section{The Poisson formula}

We now derive a Poisson formula for solutions of the inhomogeneous Dirichlet problem.

To this end, we choose a Green operator $G_{A}(\cdot, \cdot)$ for $A$ on $\mathcal{X}$, cf. 9.2.1 in [Tar95]. Given an oriented hypersurface $S \subset \mathcal{X}$, we denote by $[S]^{A}$ the kernel on $\mathcal{X} \times \mathcal{X}$ defined by

$$
\left\langle[S]^{A}, v \otimes y\right\rangle_{\mathcal{X} \times \mathcal{X}}=\int_{S} G_{A}(v, y)
$$

for all $v \in C^{\infty}\left(\mathcal{X}, \mathbb{C}^{\ell}\right)$ and $y \in C^{\infty}\left(\mathcal{X}, \mathbb{C}^{k}\right)$ whose supports meet each other in a compact set.

In particular, the kernel $[\partial \mathcal{D}]^{A}$ on $\mathcal{X} \times \mathcal{X}$ is obviously supported on the diagonal of $\partial \mathcal{D} \times \partial \mathcal{D}$.

For a function $y \in C^{\infty}\left(\overline{\mathcal{D}}, \mathbb{C}^{k}\right)$, we set

$$
\begin{aligned}
(M y)(x) & =-G A^{*}\left([\partial \mathcal{D}]^{A} y\right) \\
& =-\int_{\partial \mathcal{D}} G_{A}\left(K_{G A^{*}}(x, \cdot), y\right)
\end{aligned}
$$

when $x \in \mathcal{D}, K_{G A^{*}}$ being the Schwartz kernel of $G A^{*}$. The integral on the righthand side is well defined, for $K_{G A^{*}}$ is a $(k \times \ell)$-matrix of $C^{\infty}$ functions outside of the diagonal of $\overline{\mathcal{D}} \times \overline{\mathcal{D}}$.

Corollary 8.1. As defined above, the integral $M$ induces a continuous mapping $P$ of $W^{-s-1 / q, q}\left(\partial \mathcal{D}, \mathbb{C}^{k}\right)$ to $W^{-s, q}\left(\mathcal{D}, \mathbb{C}^{k}\right)$ such that $P t(y)=M y$. Moreover, for each weak solution $y$ of (7.2),

$$
y=H y+G \Delta y+P t(y) .
$$

Proof. Let $y \in W^{-s^{\prime}, q}\left(\mathcal{D}, \mathbb{C}^{k}\right)$ be a weak solution to the problem (7.2). From Theorem 7.2 we deduce that $y \in W^{-s, q}\left(\mathcal{D}, \mathbb{C}^{k}\right)$ satisfies $(7.2)$ in a strong sense. More precisely, there exists a sequence $y_{\nu} \in C^{\infty}\left(\overline{\mathcal{D}}, \mathbb{C}^{k}\right)$ which approximates $y$ in $W^{-s, q}\left(\mathcal{D}, \mathbb{C}^{k}\right)$, such that $t\left(y_{\nu}\right) \rightarrow t(y)$ and $\Delta y_{\nu} \rightarrow \Delta y$ in the relevant norms. We now set

$$
\begin{aligned}
P y_{0} & :=\lim _{\nu \rightarrow \infty}\left(y_{\nu}-H y_{\nu}-G\left(\Delta y_{\nu}\right)\right) \\
& =y-H y-G(\Delta y),
\end{aligned}
$$

the limit existing in $W^{-s, q}\left(\mathcal{D}, \mathbb{C}^{k}\right)$ by Theorem 6.2. Moreover, it is independent of the particular choice of $y$ with a well-defined $\Delta y$ and $t(y)=y_{0}$, which is again due to Theorem 6.2.

It is obvious that $y_{0} \mapsto P y_{0}$ is a continuous mapping of $W^{-s-1 / q, q}\left(\partial \mathcal{D}, \mathbb{C}^{k}\right)$ to $W^{-s, q}\left(\mathcal{D}, \mathbb{C}^{k}\right)$, and it remains to prove that it agrees with $-G A^{*}\left([\partial \mathcal{D}]^{A} y\right)$ in the interior of $\mathcal{D}$. 
If $z \in C^{\infty}\left(\overline{\mathcal{D}}, \mathbb{C}^{k}\right)$ has compact support in the interior of $\mathcal{D}$ then by Theorem 6.2 we get

$$
\begin{aligned}
\left(P y_{0}, z\right) & =(y, z)-(y, H z)-(\Delta y, G z) \\
& =(y, z-H z-\Delta(G z))-\int_{\partial \mathcal{D}}(t(y), n(A G z))_{x} d s \\
& =-\int_{\partial \mathcal{D}}(t(y), n(A G z))_{x} d s,
\end{aligned}
$$

for $t(G z)=0$. The right-hand side here just amounts to $\left(-G A^{*}\left([\partial \mathcal{D}]^{A} y\right), z\right)$, provided that $y$ is smooth enough.

From (8.2) it easily follows that $P y_{0}$ is the unique solution to the Dirichlet problem

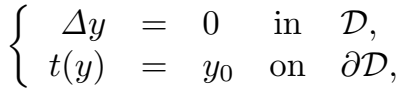

which is orthogonal to $\mathcal{H}(\mathcal{D})$. We call $P y_{0}$ the Poisson integral of $y_{0}$. By Theorem 7.2 ,

$$
\left\|P u_{0}\right\|_{-s, q} \leq c\left\|y_{0}\right\|_{W^{-s-1 / q, q}\left(\partial \mathcal{D}, \mathbb{C}^{k}\right)}
$$

with $c$ a constant independent of $y_{0}$.

\section{Nonlinear perturbation}

Since our study of the Dirichlet problem is motivated by applications to the Cauchy problem (3.1), we assume from now on that the equation $A y=0$ has no non-trivial solutions on $\mathcal{X}$ with support in $\overline{\mathcal{D}}$. Hence it follows that $\mathcal{H}(\mathcal{D})=\{0\}$, and so $H=0$.

On having a reasonable theory for the Dirichlet problem (7.2) we are in a position to study its nonlinear perturbations, i.e.,

$$
\begin{aligned}
\Delta y & =\varepsilon A^{*} f\left(x, y, y^{\prime}\right) & \text { in } \mathcal{D}, \\
t(y) & =y_{0} & \text { on } \partial \mathcal{D},
\end{aligned}
$$

cf. (4.1). We are looking for a solution $y \in W^{1, q}\left(\mathcal{D}, \mathbb{C}^{k}\right)$ to this problem, assuming $y_{0} \in W^{1-1 / q, q}\left(\partial \mathcal{D}, \mathbb{C}^{k}\right)$.

Lemma 9.1. Problem (9.1) is equivalent to the integral equation

$$
y=P y_{0}+\varepsilon G A^{*} f\left(x, y, y^{\prime}\right) .
$$

Proof. If $y$ satisfies (9.2), then $y$ is a solution to (9.1), as follows from the properties of $G$ and $P$. Conversely, applying the Green operator $G$ to both sides of the differential equation in (9.1) and using (8.1) under the Dirichlet condition $t(y)=y_{0}$, we arrive at (9.2).

The solvability of (9.2) follows by successive approximations, once we prove that $T(y):=P y_{0}+\varepsilon G A^{*} f\left(x, y, y^{\prime}\right)$ is a contraction operator in $W^{1, q}\left(\mathcal{D}, \mathbb{C}^{k}\right)$.

\section{Lemma 9.2. If}

$$
|\varepsilon|<\left(\left\|G A^{*}\right\|_{\mathcal{L}\left(L^{q}\left(\mathcal{D}, \mathbb{C}^{\ell}\right), W^{1, q}\left(\mathcal{D}, \mathbb{C}^{k}\right)\right)}\left(\left(\Lambda_{y} f\right)^{q^{\prime}}+\left(\Lambda_{p} f\right)^{q^{\prime}}\right)^{1 / q^{\prime}}\right)^{-1},
$$

then $T$ is a contraction operator in $W^{1, q}\left(\mathcal{D}, \mathbb{C}^{k}\right)$.

Proof. By Theorem 6.2, for each $1<q<\infty$ the operator $G A^{*}$ maps $L^{q}\left(\mathcal{D}, \mathbb{C}^{\ell}\right)$ continuously to $W^{1, q}\left(\mathcal{D}, \mathbb{C}^{k}\right)$. Since

$$
T(y)-T(z)=\varepsilon G A^{*}\left(f\left(x, y, y^{\prime}\right)-f\left(x, z, z^{\prime}\right)\right),
$$


it follows that

$$
\begin{aligned}
\|T(y)-T(z)\|_{W^{1, q}\left(\mathcal{D}, \mathbb{C}^{k}\right)} & \leq|\varepsilon|\left\|G A^{*}\right\|_{\mathcal{L}\left(L^{q}\left(\mathcal{D}, \mathbb{C}^{\ell}\right), W^{1, q}\left(\mathcal{D}, \mathbb{C}^{k}\right)\right)} \\
& \times\left\|f\left(x, y, y^{\prime}\right)-f\left(x, z, z^{\prime}\right)\right\|_{L^{q}\left(\mathcal{D}, \mathbb{C}^{\ell}\right)}
\end{aligned}
$$

for all $y, z \in W^{1, q}\left(\mathcal{D}, \mathbb{C}^{k}\right)$. On the other hand, an easy computation shows that

$$
\begin{aligned}
\left\|f\left(x, y, y^{\prime}\right)-f\left(x, z, z^{\prime}\right)\right\|_{L^{q}\left(\mathcal{D}, \mathbb{C}^{\ell}\right)} & \leq\left(\int_{\mathcal{D}}\left(\Lambda_{y} f|y-z|+\Lambda_{p} f\left|y^{\prime}-z^{\prime}\right|\right)^{q} d x\right)^{1 / q} \\
& \leq\left(\left(\Lambda_{y} f\right)^{q^{\prime}}+\left(\Lambda_{p} f\right)^{q^{\prime}}\right)^{1 / q^{\prime}}\|y-z\|_{W^{1, q}\left(\mathcal{D}, \mathbb{C}^{k}\right)}
\end{aligned}
$$

which implies the desired conclusion.

Let us state the final result.

Theorem 9.3. Let $1<q<\infty$. If $\varepsilon$ satisfies the estimate of Lemma 9.2, then the Dirichlet problem (9.1) has a unique solution in $W^{1, q}\left(\mathcal{D}, \mathbb{C}^{k}\right)$ for each data $y_{0} \in W^{1-1 / q, q}\left(\partial \mathcal{D}, \mathbb{C}^{k}\right)$.

Proof. By the fixed point theorem of Banach, the recurrent sequence

$$
y_{k+1}:=P y_{0}+\varepsilon G A^{*} f\left(x, y_{k}, y_{k}^{\prime}\right)
$$

converges in $W^{1, q}\left(\mathcal{D}, \mathbb{C}^{k}\right)$ to the unique solution of $(9.2)$ as a geometric series, provided that $\varepsilon$ is small enough. Moreover, the start approximation can be chosen arbitrarily.

Were $f\left(x, y, y^{\prime}\right)$ independent of $y^{\prime}$, one could consider the iterations of $T$ in the space $L^{q}\left(\mathcal{D}, \mathbb{C}^{k}\right)$. Since the kernel of $G A^{*}$ is locally integrable over $\overline{\mathcal{D}}, T$ would be a contraction operator for $\mathcal{D}$ small enough. The recurrent sequence $y_{k+1}$ would actually converge in $W^{1, q}\left(\mathcal{D}, \mathbb{C}^{k}\right)$, for $T$ would map $L^{q}\left(\mathcal{D}, \mathbb{C}^{k}\right)$ continuously to $W^{1, q}\left(\mathcal{D}, \mathbb{C}^{k}\right)$.

\section{The Cauchy problem Revisited}

Throughout this section, we assume that $A^{*} u=0$ has no non-trivial solutions on $\mathcal{X}$ with support in $\overline{\mathcal{D}}$.

Given any $y_{0} \in W^{1-1 / q, q}\left(\partial \mathcal{D}, \mathbb{C}^{k}\right)$, we denote by $y=\mathcal{R}\left(x ; y_{0}\right)$ the solution of (9.1) guaranteed by Theorem 9.3 .

Theorem 9.3 applies directly to the Cauchy problem (3.1) only in the case where $\mathcal{S}$ is the whole boundary of $\mathcal{D}$.

Corollary 10.1. Suppose that $y_{0} \in W^{1-1 / q, q}\left(\partial \mathcal{D}, \mathbb{C}^{k}\right)$, where $1<q<\infty$. In order that there be a function $y \in W^{1, q}\left(\mathcal{D}, \mathbb{C}^{k}\right)$ satisfying $A y=\varepsilon f\left(x, y, y^{\prime}\right)$ in $\mathcal{D}$ and $y=y_{0}$ on $\partial \mathcal{D}$, it is necessary and sufficient that $A \mathcal{R}\left(x ; y_{0}\right)=\varepsilon f\left(x, y_{0}, \mathcal{R}\left(x ; y_{0}\right)^{\prime}\right)$ on $\partial \mathcal{D}$.

Proof. If $y \in W^{1, q}\left(\mathcal{D}, \mathbb{C}^{k}\right)$ satisfies $A y=\varepsilon f\left(x, y, y^{\prime}\right)$ in $\mathcal{D}$ and $y=y_{0}$ on $\partial \mathcal{D}$, then $y=\mathcal{R}\left(x ; y_{0}\right)$ by Theorem 9.3. Hence $A \mathcal{R}\left(x ; y_{0}\right)=\varepsilon f\left(x, y_{0}, \mathcal{R}\left(x ; y_{0}\right)^{\prime}\right)$ on $\partial \mathcal{D}$. Conversely, if $A \mathcal{R}\left(x ; y_{0}\right)=\varepsilon f\left(x, y_{0}, \mathcal{R}\left(x ; y_{0}\right)^{\prime}\right)$ on $\partial \mathcal{D}$, then this equality holds everywhere in $\mathcal{D}$, which is due to Theorem 4.1. Hence $y=\mathcal{R}\left(x ; y_{0}\right)$ is a solution of (3.1).

Corollary 10.1 reduces the Cauchy problem (3.1) in the case $\mathcal{S}=\partial \mathcal{D}$ to an operator equation on the boundary. As but one consequence of this reduction we mention that the Cauchy problem with data on the whole boundary is normally solvable.

If $\mathcal{S} \neq \partial \mathcal{D}$ then the Cauchy data are insufficient to uniquely solve the Dirichlet problem. What is lacking is the knowledge of $y$ on $\partial \mathcal{D} \backslash \mathcal{S}$, which may be thought of as a new unknown function $z$ with values in $\mathbb{C}^{k}$. The function $\chi_{\mathcal{S}} y_{0}+\chi_{\partial \mathcal{D} \backslash \mathcal{S}} z$ on $\partial \mathcal{D}$, which we denote simply by $y_{0} \oplus z$, must be in $W^{1-1 / q, q}\left(\partial \mathcal{D}, \mathbb{C}^{k}\right)$. Therefore, 
we require $z \in W^{1-1 / q, q}\left(\partial \mathcal{D} \backslash \mathcal{S}, \mathbb{C}^{k}\right)$ to fulfil the compatibility condition $z=y_{0}$ on $\partial \mathcal{S}$, for the functions of $W^{1-1 / q, q}\left(\partial \mathcal{D} \backslash \mathcal{S}, \mathbb{C}^{k}\right)$ possess traces on $\partial \mathcal{S}$ provided $q>2$. The solution of the non-linear Dirichlet problem with data $y_{0} \oplus z$ on $\partial \mathcal{D}$ is $y=\mathcal{R}\left(x ; y_{0} \oplus z\right)$, and we substitute this into the second boundary condition in (4.1), thus obtaining

$$
\begin{aligned}
A \mathcal{R}\left(x ; y_{0} \oplus z\right) & =\varepsilon f\left(x, y_{0} \oplus z, \mathcal{R}\left(x ; y_{0} \oplus z\right)^{\prime}\right) & & \text { on } \partial \mathcal{D} \\
z & =y_{0} & & \text { on } \partial \mathcal{S} .
\end{aligned}
$$

Corollary 10.2. Suppose that $y_{0} \in W^{1-1 / q, q}\left(\mathcal{S}, \mathbb{C}^{k}\right)$, where $1<q<\infty$. In order that there exist a solution $y \in W^{1, q}\left(\mathcal{D}, \mathbb{C}^{k}\right)$ to the Cauchy problem (3.1) it is necessary and sufficient that there be a function $z \in W^{1, q}\left(\partial \mathcal{D} \backslash \mathcal{S}, \mathbb{C}^{k}\right)$ satisfying (10.1)

Proof. The proof immediately follows from Theorem 4.1.

This corollary seems to be of no practical importance but of purely mathematical interest. It reduces the Cauchy problem in a domain $\mathcal{D}$ with data on $\mathcal{S} \subset \partial \mathcal{D}$ to a boundary problem in $\partial \mathcal{D} \backslash \mathcal{S}$ with data on $\partial \mathcal{S}$. This latter requires certainly further investigations.

\section{The Zaremba Problem}

Among the functions $u: \mathcal{D} \rightarrow \mathbb{C}^{\ell}$ that are continuous up to the boundary one can specify those having the zero Cauchy data on $\partial \mathcal{D} \backslash \mathcal{S}$ relative to the differential operator $A^{*}$. We write $n(u)=0$ on $\partial \mathcal{D} \backslash \mathcal{S}$ for these functions, which precisely means that $\sigma(A)(x, \nu)^{*} u=0$ on $\partial \mathcal{D} \backslash \mathcal{S}$, where $\sigma(A)(x, \nu)$ is the principal symbol of $A$ evaluated at the unit outward normal vector $\nu$ of the boundary. For functions $u \in L^{q}\left(\mathcal{D}, \mathbb{C}^{\ell}\right)$, the equality $n(u)=0$ on $\partial \mathcal{D} \backslash \mathcal{S}$ makes still sense if one employs negative norms.

The mixed boundary problem

$$
\begin{aligned}
& \Delta y=\varepsilon A^{*} f\left(x, y, y^{\prime}\right) \quad \text { in } \mathcal{D}, \\
& y=y_{0} \quad \text { on } \mathcal{S} \text {, } \\
& n(A y)=\varepsilon n\left(f\left(x, y, y^{\prime}\right)\right) \text { on } \partial \mathcal{D} \backslash \mathcal{S} \text {, }
\end{aligned}
$$

the last equality meaning $n\left(A y-\varepsilon f\left(x, y, y^{\prime}\right)\right)=0$ on $\partial \mathcal{D} \backslash \mathcal{S}$, generalises the classical Zaremba problem, see [Zar10]. Obviously, problems (11.1) and (4.1) have much in common.

Note that if $\mathcal{S}=\partial \mathcal{D}$ then (11.1) just amounts to the Dirichlet problem considered in $\S 5$.

If $y \in W^{1, q}\left(\mathcal{D}, \mathbb{C}^{k}\right)$ is a solution to the Cauchy problem (3.1), then it satisfies (11.1) as well. The advantage of using (11.1) lies in the fact that for a wide class of (necessarily overdetermined, unless $n=1$ ) differential operators $A$ the unperturbed (i.e., corresponding to $\varepsilon=0$ ) mixed boundary value problem (11.1) is elliptic. For $q=2$ it can be studied within pseudodifferential calculus on manifolds with edges, cf. [Sch98].

The Hodge parametrix of the unperturbed problem (11.1) allows one to reduce the perturbed problem to an integral equation similar to (9.2). In contrast to Corollary 10.2 we then obtain solvability conditions of (3.1) like those of Corollary 10.1. More precisely, $n\left(A \mathcal{R}\left(x ; y_{0}\right)-\varepsilon f\left(x, y_{0}, \mathcal{R}\left(x ; y_{0}\right)^{\prime}\right)\right)=0$ on $\mathcal{S}$, where $\mathcal{R}$ is the resolution operator for (11.1).

This shows that if $n \circ A$ is a complementary boundary condition for $A^{*} A$, then the Cauchy problem (3.1) is normally solvable for any non-empty open piece $\mathcal{S} \subset \partial \mathcal{D}$ with smooth boundary.

Remark 11.1. The mixed boundary value problems in $\mathcal{D}$ with interface surface $\partial \mathcal{S}$ are usually studied in weighted Sobolev spaces, the weights being powers of the distance to $\partial \mathcal{S}$. 
The operator

$$
y_{0} \mapsto n\left(A \mathcal{R}\left(x ; y_{0}\right)-\varepsilon f\left(x, y_{0}, \mathcal{R}\left(x ; y_{0}\right)^{\prime}\right)\right)
$$

on $\mathcal{S}$ is a far-reaching generalisation of the so-called 'Dirichlet to Neumann' operator, cf. [Tay96, $12 \mathrm{C}]$.

This latter $\mathcal{N}$ corresponds to the linear case $\varepsilon=0$ with $A=\nabla$ being the gradient operator and $\mathcal{S}=\partial \mathcal{D}$. The operator $\mathcal{N}$ is known to be a negative semidefinite, selfadjoint, and also elliptic pseudodifferential operator of order 1 on the boundary. It actually coincides with the negative square root of the Laplace-Beltrami operator of $\partial \mathcal{D}$ modulo zero order operators, see $i b i d$. If the boundary is connected, then the null space of $\mathcal{N}$ consists of all constant functions on $\partial \mathcal{D}$, which agrees with Corollary 10.1 .

\section{LINEARISABLE PROBLEMS}

In this section we compare the results obtained above with those coming from the linear theory under transformations of variables, see [Tar95]. Namely, we consider the Cauchy problem for a Dirac system ${ }^{1} D w=0$ in a bounded domain $\mathcal{G} \subset \mathbb{R}^{n}$ with smooth boundary, the data $w=w_{0}$ being given on an open part $\mathcal{T}$ of $\partial \mathcal{G}$, and a global transformation

$$
\left\{\begin{array}{l}
x=X(z, w) \\
y=Y(z, w)
\end{array}\right.
$$

of both independent and dependent variables defined in a neighbourhood of $\overline{\mathcal{G}} \times \mathbb{C}^{k}$. We require $(12.1)$ to be a diffeomorphism and write $z=Z(x, y), w=W(x, y)$ for the inverse transformation.

Each $\mathbb{C}^{k}$-valued function $w=w(z)$ uniquely determines a function $y=y(x)$ with values in $\mathbb{C}^{k}$, and conversely. Indeed, pick $x \in \mathcal{X}$. Using $x=X(z, w(z))$ we find $z$, and hence $y=Y(z, w(z))$ in $\mathbb{C}^{k}$.

By the chain rule,

$$
w_{z_{j}}^{\prime}=\left(W_{x}^{\prime}+W_{y}^{\prime} y_{x}^{\prime}\right) X_{z_{j}}^{\prime}
$$

and so $D w=\sum_{j=1}^{n} A_{j} w_{z_{j}}^{\prime}$ transforms into

$$
\sum_{j=1}^{n} D X_{j} W_{y}^{\prime} y_{x_{j}}^{\prime}+\left(A_{1} W_{x}^{\prime} X_{z_{1}}^{\prime}+\ldots+A_{n} W_{x}^{\prime} X_{z_{n}}^{\prime}\right),
$$

$D$ applies to $X_{j}$ in an obvious way. This is quasilinear differential operator on an open set $\mathcal{X} \subset \mathbb{R}^{n}$. We freeze the coefficients of the principal part at some fixed function $y=v(x)$ and set

$$
A y=\left.\sum_{j=1}^{n}\left(D X_{j} W_{y}^{\prime}\right)\right|_{y=v} y_{x_{j}}^{\prime}
$$

thus obtaining a first order linear differential operator on $\mathcal{X}$ whose principal symbol has rank $k$ at each point $(x, \xi) \in T^{*} \mathcal{X}$ with $\xi \neq 0$.

Suppose $\mathcal{G}$ transforms into a domain $\mathcal{D} \Subset \mathcal{X}$. The condition $W(x, y)=w_{0}$ on the set $\mathcal{T} \subset \partial \mathcal{G}$ determines a unique function $y_{0}$ on an open part $\mathcal{S}$ of $\partial \mathcal{D}$ with values in $\mathbb{C}^{k}$. Hence, the Cauchy problem for solutions of $D w=0$ in $\mathcal{G}$ is equivalent to the Cauchy problem

$$
\begin{array}{rlr}
A y & =f\left(x, y, y^{\prime}\right) & \text { in } \mathcal{D} \\
y & =y_{0} & \text { on } \mathcal{S}
\end{array}
$$

\footnotetext{
${ }^{1}$ An $(\ell \times k)$-matrix $D$ of first order differential operators is said to be a (generalised) Dirac operator if $D^{*} D=\Delta E_{k}$, where $\Delta$ is the Laplace-Beltrami operator and $E_{k}$ the identity $(k \times k)$ matrix.
} 
where

$f\left(x, y, y^{\prime}\right)=-\sum_{j=1}^{n}\left(D X_{j} W_{y}^{\prime}-\left.D X_{j} W_{y}^{\prime}\right|_{y=v}\right) y_{x_{j}}^{\prime}-\left(A_{1} W_{x}^{\prime} X_{z_{1}}^{\prime}+\ldots+A_{n} W_{x}^{\prime} X_{z_{n}}^{\prime}\right)$, cf. (3.1).

For regularisation of the Cauchy problem $D w=0$ in $\mathcal{G}$ and $w=w_{0}$ on $\mathcal{T}$ with $w_{0}$ integrable over $\mathcal{T}$ there are the so-called Carleman formulas. These are of the form

$$
w(z)=\lim _{N \rightarrow \infty} \int_{\mathcal{T}} D^{*} \mathcal{C}^{(N)}(z, \cdot)\left(\sum_{j=1}^{n} A_{j} \nu_{j}\right) w_{0} d s
$$

for $z \in \mathcal{G}$, where $\nu_{j}$ is the $j$ th component of the unit outward normal vector to $\partial \mathcal{G}$, $d s$ is the surface measure on $\partial \mathcal{G}, D^{*}$ the formal adjoint of $D$, and $\mathcal{C}^{(N)}(z, \cdot)$ is a sequence of explicitly given kernels defined on $\mathcal{G} \times \overline{\mathcal{G}}$ away from the diagonal, see [Tar95, 12.4.6]. Vice versa, the Carleman formulas yield also solvability conditions for the Cauchy problem, for if the limit on the right-hand side of (12.3) exists for each $z \in \mathcal{G}$ then it defines a function $w: \mathcal{G} \rightarrow \mathbb{C}^{k}$ satisfying $D w=0$ in $\mathcal{G}$ and $w=w_{0}$ on $\mathcal{T}$, cf. ibid.

Our next goal is to interpret formula $(12.3)$ in the coordinates $(x, y)$, i.e., for the Cauchy problem (12.2). For simplicity, we will restrict our discussion to the case where $x=X(z)$ and $y=Y(w)$ are independent of $w$ and $z$, respectively. Then, the inverse transformation is of the form $z=Z(x), w=W(y)$. Functions $w=w(z)$ and $y=y(x)$ correspond to each other under this transformation if and only if $W \circ y=w \circ Z$.

It is easy to see that (12.3) transforms into

$$
y(x)=\lim _{N \rightarrow \infty} W^{-1} \int_{\mathcal{S}} D^{*} \mathcal{C}^{(N)}(Z(x), Z(\cdot))\left(\sum_{j=1}^{n} A_{j} \nu_{j} \circ Z\right)\left|\operatorname{det} Z^{\prime}\right| W\left(y_{0}\right) d s,
$$

for $x \in \mathcal{D}$, where $Z^{\prime}$ is the Jacobi matrix of $Z$, and we use the same symbol $d s$ to designate the surface measure on $\partial \mathcal{D}$. This formula is of general character and makes sense for diverse linearisable systems $A y=\varepsilon f\left(x, y, y^{\prime}\right), A$ being of injective symbol.

It should be noted that (12.4) may fail for some transformations of dependent variables $w=W(y)$. As applications show, the approximating functions $T^{(N)} W\left(y_{0}\right)$ in

$$
y=\lim _{N \rightarrow \infty} W^{-1}\left(T^{(N)} W\left(y_{0}\right)\right)
$$

can leave the domain of $W^{-1}$. 


\section{REFERENCES}

[Dez80] Dezin, A. A., General Questions of the Theory of Boundary Value Problems, Nauka, Moscow, 1980.

[Had27] Hadamard, J., Quelques cas d'impossibilité du problème de Cauchy, In: Memorial N. I. Lobachevsky 2 (1927), 163-176.

[KMF91] Kozlov, V. A., Maz'Ya, V. G., and Fomin, A. V., An iterative method for solving the Cauchy problem for elliptic equations, Comput. Maths. Math. Phys. 31 (1991), no. 1, $45-52$.

[Kry69] Krylov, A. L., The Cauchy problem for Laplace's equation in the complex domain, Soviet Math. Dokl. 10 (1969), 1184-1187.

[Mas68] MasLov, V. P., The existence of a solution of an ill-posed problem is equivalent to the convergence of a regularization process, Uspekhi Mat. Nauk 23 (1968), no. 3, 183-184.

[Mor58] Morrey, C., On the analyticity of the solutions of analytic non-linear elliptic systems of partial differential equations. II. Analyticity at the boundary, Amer. J. Math. 80 (1958), 219-237.

[Pal68] Palais, R., Foundations of global non-linear analysis, Benjamin, New York, 1968.

[Pry81] Pryde, A. J., Second order elliptic equations with mixed boundary conditions, J. Math. Anal. Appl. 80 (1981), no. 1, 203-244.

[Roi96] Roitberg, YA. A., Elliptic Boundary Value Problems in Generalized Functions, Kluwer Academic Publishers, Dordrecht NL, 1996.

[SST00] Schulze, B.-W., Shlapunov, A., and N. Tarkhanov, N., Green integrals on manifolds with cracks, Annals of Global Analysis and Geometry 24 (2003), no. 2, 131-160.

[Sch98] Schulze, B.-W., Boundary Value Problems and Singular Pseudo-Differential Operators, J. Wiley, Chichester, 1998.

[Sch60] Schechter, M., Negative norms and boundary problems, Ann. Math. 72 (1960), no. 3, 581-593.

[ST03] Shlapunov, A., and Tarkhanov, N., Duality by reproducing kernels, IJMMS 6 (2003), January, 327-395.

[ST05] Shlapunov, A., and Tarkhanov, N., Mixed problems with a parameter, Russian J. Math. Phys. 12 (2005), no. 1, 97-119.

[Tar95] Tarkhanov, N., The Cauchy Problem for Solutions of Elliptic Equations, Akademie Verlag, Berlin, 1995.

[Tay96] TaYlor, M. E., Partial Differential Equations II, Springer-Verlag, New York, 1996.

[TA79] Tikhonov, A. N. and Arsenin, V. Ya., Methods of Solution of Ill-Posed Problems, Nauka, Moscow, 1979.

[VS00] L. R. Volevich, L. R., and ShiRikyan, A. R., Stable and unstable manifolds for nonlinear elliptic equations with parameter, Trans. Moscow Math. Soc. 61 (2000), 97-138.

[Zar10] Zaremba, S., Sur un problème mixte à l'équation de Laplace, Bull. Intern. Acad. Sci. Cracovie ? (1910), 314-344.

(Ibrahim Ly) Institut für Mathematik, Universität Potsdam, Postfach 6015 53, 14415 Potsdam, Germany

E-mail address: ?@?

(Nikolai Tarkhanov) Institut für Mathematik, Universität Potsdam, Postfach 6015 53, 14415 Potsdam, Germany

E-mail address: tarkhanov@math.uni-potsdam.de 